\title{
Teoria da identidade social aplicada ao jogo de autorregulação dos processos de trocas sociais baseado em sistemas multiagente
}

\author{
Jader de Freitas Saldanha ${ }^{1}$ \\ Diana Adamatti ${ }^{1}$ \\ Graçaliz Dimuro ${ }^{1}$
}

\begin{abstract}
Resumo: A Teoria da Identidade Social enfatiza a dimensão social do comportamento individual e grupal, ao postular que o indivíduo é moldado pela sociedade e pela cultura. Desenvolvido por Macedo, o Jogo de Autorregulação dos Processos de Trocas Sociais, apresenta agentes artificiais comunicando-se pelas Trocas Sociais levando em conta suas diferentes estratégias de jogo até que o sistema atinja um equilíbrio em relação as trocas. A metodologia de desenvolvimento desse trabalho baseou-se na revisão de literatura sobre os conceitos necessários envolvidos nessa pesquisa, bem como o olhar de trabalhos da comunidade científica sobre a Teoria da Identidade Social e os Sistemas Multiagente e então a elaboração de uma proposta inicial. Como objetivo geral tem-se: desenvolver um modelo de simulação que integre os conceitos da Teoria da Identidade Social no âmbito do Jogo de Autorregulação dos Processos de Trocas Sociais. Como objetivos específicos: identificar em trabalhos relacionados contribuições para o desenvolvimento; elaborar uma proposta inicial para a análise de simulação multiagente. Espera-se que essa pesquisa contribua na análise de características emergentes do comportamento humano em ambientes artificiais que até então não foram investigadas coletivamente.
\end{abstract}

Palavras-chave: Simulação Social, Psicologia Social, Teoria da Identidade Social

\begin{abstract}
The Social Identity Theory emphasizes the individual and group social behavior by saying that the individual is build up by society and culture. Developed by Macedo, The Selfregulating Game of Social Exchanges, presents artificial agents communicating by Social Exchanges taking into account their different game strategies until the systems arise the equilibrium by means of social exchanges. This work was based on the literature review about the fundamental concepts used in this research, as the overview in the scienfitic community work on social identity theory and multi-agent systems and the initial proposal. As the main goal: develop a simulation model that mixes Social Identity Theory in the Self-regulating Game of Social Exchanges. The specific objectives: identify in related work contributions for development; An initial proposal for multi-agent simulation. It is expected that this research works in favor of emerging characteristics of human behavior in artificial enviroments that was not until now studied collectively.
\end{abstract}

Keywords: Social Simulation. Social Psycology. Social Identity Theory.

\section{Introdução}

Em um modelo de interação social real entre seres humanos, discriminações sociais intergrupais acontecem. [1] [2] por meio de experimentos comprovaram que por uma mera categorização social dentro e fora de grupos, é provocado algum tipo de favorecimento a seus semelhantes. Os estudos originários de [1] e seus colaboradores trouxeram a Teoria da Identidade Social (TIS), que segundo a autora [3], procurava enfatizar a dimensão social do comportamento individual e grupal, ao postular que o indivíduo é moldado pela sociedade e pela cultura. Nesse sentido, defende que as relações intergrupais estão intimamente relacionadas a processos de identificação grupal

\footnotetext{
${ }^{1}$ Programa de Pós-Graduação em Computação (PPGComp), Universidade Federal do Rio Grande, Centro de Ciências Computacionais - C3 - Rio Grande (RS) - Brasil

\{jaderdsaldanha@furg.br, dianaada@furg.br,gracaliz@gmail.com\}
}

http://dx.doi.org/10.5335/rbca.v9i1.6728

Revista Brasileira de Computação Aplicada (ISSN 2176-6649), Passo Fundo, v. 9, n. 1, p. 73-83, abr. 2017 
e de comparação social. A autora menciona que a TIS apoia-se em três postulados básicos: (1) o autoconceito é derivado da identificação e pertença grupal; (2) as pessoas são motivadas a manter uma autoestima positiva; (3) as pessoas estabelecem uma identidade social positiva mediante a comparação favorável de seu próprio grupo (in-group) com outros grupos sociais (out-groups). Nesse sentido, quando tal comparação não se mostra favorável ao próprio grupo, elas irão adotar diferentes estratégias para recuperar o favoritismo de seu próprio grupo, como forma de assegurar uma autoestima positiva.

Há pesquisas que elucidam trajetória na tentativa de modelagem da TIS em Sistemas Multiagentes (SMA), dentre elas [4] [5] [6] [7] [8] [9] [10], que ressaltam a importância de características da psicologia social em agentes. Na literatura, Trocas Sociais (TS) em SMA são objeto de estudo em diversos contextos, nos quais as relações sociais são interpretadas como TS ([11] [12] [13] [14] [15]). Como investigado por [13], um problema fundamental discutido na literatura é a regulação das TS, por exemplo, a emergência de trocas equilibradas ao longo do tempo levando ao equilíbrio social e/ou comportamento de equilíbrio/justiça. Desenvolvido por [13], o JAPTS (Jogo de Autorregulação dos Processos de Trocas), é um jogo onde agentes artificiais interagem por um protocolo de comunicação levando em consideração suas diferentes estratégias (egoísta, altruísta e racional), ao longo do tempo os agentes evoluem suas estratégias até que se chegue a um ponto de equilíbrio das trocas sociais. Até o momento, uma análise a luz do comportamento dos agentes (e da sociedade) levando em conta estudos da TIS não foi desenvolvida. Portanto, a metodologia de desenvolvimento desse trabalho baseou-se na revisão de literatura sobre os conceitos necessários envolvidos nessa pesquisa, bem como o olhar de trabalhos da comunidade científica sobre a Teoria da Identidade Social e os Sistemas Multiagente e então a elaboração de uma proposta inicial. Como objetivo geral tem-se: desenvolver um modelo de simulação que integre os conceitos da Teoria da Identidade Social no âmbito do Jogo de Autorregulação dos Processos de Trocas Sociais. Como objetivos específicos: identificar em trabalhos relacionados contribuições para o desenvolvimento; elaborar uma proposta inicial para a análise de simulação multiagente. Além disso, pela análise da viabilidade de implementação em uma ferramenta multiagente, foi desenvolvido um modelo preliminar do experimento original de [2] em [16]. Essas estruturas de dados serão utilizadas em trabalhos futuros.

O trabalho está organizado da seguinte maneira: na seção 2 é apresentado o referencial teórico para esta pesquisa, na seção 3 os trabalhos relacionados aos temas, na seção 4 uma proposta de um classificador de interação da sociedade do JAPTS baseado em conceitos de grafos e no framework computacional identificado nos trabalhos relacionados sobre TIS e SMA. Na seção 5 as conclusões parciais, na 6 os agradecimentos e na 7 as referências.

\section{Referencial teórico}

\subsection{Teoria da identidade social}

[3] contextualiza a teoria da identidade social ao surgir na literatura sociopsicológica com Henri Tajfel, da Universidade de Bristol, na Inglaterra, e seus colaboradores [2] [1] [17] [18], que procuraram enfatizar a dimensão social do comportamento individual e grupal, ao postularem que o indivíduo é moldado pela sociedade e pela cultura. Nesse sentido, defendem que as relações intergrupais estão intimamente relacionadas a processos de identificação grupal e de comparação social.

Segundo a autora a teoria da identidade social apoia-se em três postulados básicos:

1. O autoconceito é derivado da identificação e pertença grupal;

2. As pessoas são motivadas a manter uma autoestima positiva;

3. As pessoas estabelecem uma identidade social positiva mediante a comparação favorável de seu próprio grupo (in-group) com outros grupos sociais (out-groups).

Nesse sentido, quando tal comparação não se mostra favorável ao próprio grupo, elas irão adotar diferentes estratégias para recuperar o favoritismo de seu próprio grupo, como forma de assegurar uma autoestima positiva.

As primeiras demonstrações sobre a identificação social e o favoritismo do próprio grupo foram realizadas por [2] [1], mediante o uso de experimentos adotando a técnica do paradigma dos grupos mínimos, em que as 
pessoas eram arbitrariamente assinaladas a grupos com os quais não possuíam nenhuma identificação anterior e, mesmo assim, tendiam a manter a vantagem do próprio grupo. Desde então, o referido princípio tem sido objeto de inúmeras verificações empíricas, dentro e fora do laboratório. Tais estudos têm consistentemente apontado que a força da identificação com o próprio grupo e a necessidade de manter uma autoestima positiva encontram-se na base das relações intergupais, ao provocarem o favoritismo do próprio grupo e as atitudes discriminatórias daí decorrentes [3] [19].

A teoria da identidade social, em suas múltiplas vertentes, pode ser vista, portanto, como uma abordagem que, nos últimos 30 anos, vem procurando elucidar o papel desempenhado pelo autoconceito nos processos e relações intergrupais, mediante a articulação de fenômenos de natureza sociocognitiva, motivacional e macrossocial que permeiam a vida coletiva. Inicialmente surgida na Europa, ela tem sido adotada cada vez mais como referencial por pesquisadores de diversas partes do mundo, incluindo-se aí muitos psicólogos norte-americanos, podendo ser considerada atualmente uma das mais significativas teorias para a análise das relações entre o indivíduo e o grupo. Nesse sentido, ela vem sendo utilizada mais recentemente não apenas no estudo das relações intergrupais, mas também na investigação da autocategorização e de vários processos grupais, como a coesão, a liderança, a influência social etc. No entanto, ela continua sem resolver um de seus principais desafios, qual seja, promover a maior compreensão dos aspectos afetivos que se encontram subjacentes às formas mais hostis e destrutivas de comportamento intergrupal [3] [19] [20].

\subsection{Jogo de autorregulaçãos dos processos de trocas sociais}

Como observado por [13], as TS em SMA são objeto de estudo em diversos contextos, onde as relações sociais são interpretadas como TS, um dos problemas fundamentais estudado é a regulação das TS, ou seja, a emergência de trocas equilibradas ao longo do tempo levando a um equilíbrio social e/ou comportamento de equilíbrio/justiça. Em [13], é proposta uma abordagem ao problema da autorregulação das TS, baseada na Teoria dos Jogos. O modelo JAPTS, na sua versão evolutiva e espacial, onde os agentes são organizados em uma rede complexa e podem evoluir suas diferentes estratégias de troca social. No modelo desenvolvido por [13] é considerado um conjunto de agentes heterogêneos conectados por uma rede complexa, onde a partir da evolução de suas estratégias procuram melhorar seu valor de adaptação definido como fitness. Pelos conceitos originais elaborados por [21], as TS são interações sociais definidas como trocas de serviços entre pares de agentes e, paralelamente, existe a avaliação dessas trocas pelos próprios agentes, gerando valores materiais (o valor do investimento para a realização de um serviço e/ou o valor de satisfação para recebê-lo) e valores virtuais (débito e crédito, os quais contribuem no registro de processos de troca incompletos).

\subsubsection{Estutura das trocas sociais}

Definido em [13], os valores materiais reais, são gerados através da avaliação de trocas imediatas, ações concretas podendo representar sentimentos como gratidão, admiração, entre outros. Já os valores virtuais são gerados através de trocas postergadas, não são expressos de forma explícita, ficam armazenados na consciência dos indivíduos e, por isso, podem ser percebidos em longo prazo, como uma forma de compromisso com as trocas ocorridas no passado. [13] apresenta um exemplo: sejam dois colegas $a$ e $b$ em algum ambiente de trabalho. O colega $a$ tem uma reunião na escola de seu filho, quer ir, mas não pode devido as tarefas que precisa terminar relacionado a seu trabalho. O colega $b$ percebendo tal situação se oferece (oferta) a terminar as atividades do colega $a$ para que este possa ir a reunião. Observando o exemplo, a avaliação do indivíduo $a$ em relação a oferta realizada pelo indivíduo $b$ e esta oferta, são valores materiais gerados através da troca imediata. Considerando que a troca se realiza, esta oferta/ação de $b$ concreta pode criar o surgimento de sentimento de gratidão de $a$ com $b$. Como valores virtuais, implicitamente se armazena na consciência de $a$ uma dívida futura, compromisso com a troca ocorrida no passado com $b$ e para $b$ um crédito, o qual pode solicitar alguma tarefa para $a$ lembrando da troca no passado. Para Piaget, uma troca social sempre envolve pelo menos dois indivíduos $(i$ e $j$ ) e pode ser executada através de dois estágios de trocas. A figura 1 sumariza o exemplo.

Na primeira etapa o agente $i$ realiza uma oferta para o agente $j$. O agente $j$ tem uma satisfação $s_{j}$ referente a oferta $r_{i}$ do agente $i$. O agente $j$ gera um valor de reconhecimento $t_{j}$ em relação a satisfação causada pela oferta realizada por $i$. Finalizando a etapa I, o indivíduo $i$ tem um crédito virtual $v_{i}$ que lhe constitui um direito. Já $j$ tem uma dívida virtual $t_{j}$ que lhe constitui uma dívida.

Revista Brasileira de Computação Aplicada (ISSN 2176-6649), Passo Fundo, v. 9, n. 1, p. 73-83, abr. 2017 
Figura 1: Etapas de trocas entre dois agentes

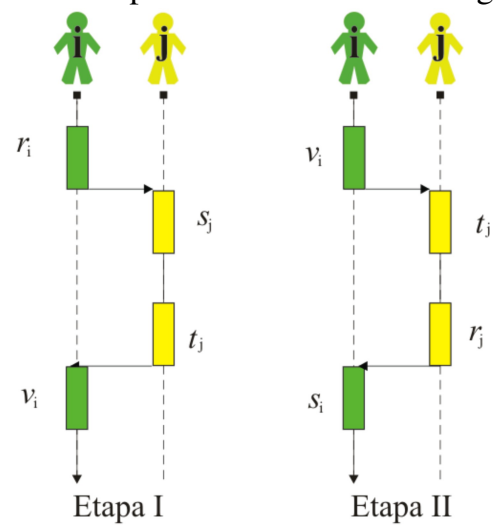

Os valores de trocas sociais gerados nesta etapa são $r_{i}, s_{j}, t_{j}$ e $v_{i}$, onde:

- $r_{i}$ é o valor do investimento do agente $i$,

- $s_{j}$ é o valor de satisfação de $j$,

- $t_{j}$ é o valor de débito de $j$,

- $v_{i}$ é o valor do crédito de $i$.

Considerando que $i$ cobra a dívida de $j$ em troca da oferta/investimento $r_{i}$ que realizou, então é preciso observar a segunda etapa de trocas entre $i$ e $j$. A segunda etapa de troca (Etapa II) é interpretada da seguinte forma: pagar uma dívida.

Então, na segunda etapa, $i$ cobra o serviço que foi previamente executado para $j$, e os valores relacionados a esta troca tem um significado semelhante aos da primeira etapa. $r_{i}, s_{j}, r_{j}$ e $s_{i}$ são chamados valores materiais e $t_{j}, v_{i}, t_{j}$ e $v_{i}$ são os valores virtuais.

Em [13], o modelo desenvolvido simula trocas sociais entre indivíduos. Para tal modelagem foi utilizado um sistema multiagente. Sendo assim, os indivíduos envolvidos nas trocas sociais do modelo são agentes em um SMA. Trata-se de um modelo de organização social, no qual as relações sociais são interpretadas como trocas sociais e valores de troca são colocados em uso no apoio à continuidade do desempenho das trocas sociais. A dinâmica das organizações sociais é formulada em termos de sistemas de valores de troca como ferramentas para a organização de sistemas multiagentes. Sistemas de valores de troca são definidos com base na teoria da troca social, desenvolvidos por Piaget. Inicialmente, foi proposto por [22] um modelo de interações entre agentes com base na teoria de trocas sociais de Piaget. Posteriormente, os trabalhos na literatura apresentam mecanismos de regulação de trocas.

\section{Trabalhos relacionados}

A Teoria da Identidade Social é altamente influente nas ciências sociais e desenvolver um modelo formal dela é um objetivo grandioso. As literaturas de áreas de ciências sociais e computacionais apresentam um grande número de tentativas de formalizar (ao menos partes da) TIS utilizando modelos baseados em agentes, dentre outros modelos não necessariamente baseado em agentes. Alguns exemplos incluem [4] [6] [5] [7] [8] [9] [10]. Contudo, seu uso aliado a teoria das trocas sociais, ainda é algo inédito em termos de pesquisa. A seguir apresentam-se alguns trabalhos, a intenção é analisar quais destes poderão ser úteis ao estender o modelo do JAPTS.

O trabalho de [23] elucida a modelagem da identidade social, contextualizando trabalhos passados e questões relevantes na modelagem sociocultural. Segundo o autor, muito dos conflitos políticos atuais são baseados em identidades sociais diferentes, e os lados são desenhados pelas visões como etnia, religião, linhas ideológicas etc. 
Os esforços da modelagem sociocultural precisam incorporar dinâmicas sociais realísticas que são baseadas na literatura acadêmica e construída com base em trabalhos anteriores. O artigo revisa quatro esforços de modelagem: SCIPR, Salzarulo Metacontrast model, PS-I e Silas. Cada um foi analisado baseado na mistura de identidades descendentes (permanentes, herdadas) e flexíveis, em como cada uma lida com a mudança saliente usando o modelo de Turner: Acessibilidade x Fit. Enquanto cada indivíduo pode ter múltiplas identidades de múltiplos tipos, a importância dessas identidades podem mudar radicalmente de uma circunstancia para outra. Os autores dizem que entender quando identidades particulares são salientes é uma capacidade crítica. Foram usados os conceitos de Acessibilidade e fit, retirados dos trabalhos de [24] [25] [26] como uma forma de pensar sobre diferenças na saliência Acessibilidade se refere a facilidade de recuperação de uma identidade a mente. Fit é o grau no qual um contexto particular ativa identidades particulares. Eventos atuais podem fortemente interagir com identidade particulares. E como cada uma utiliza dados para validação. O autor apresenta uma tabela analisando os trabalhos de [6] [27] [4] e SILAS, que segundo o autor [23], está em fase de desenvolvimento. A tabela 1 apresenta a sumarização dos modelos. Baseado na ideia dos autores [23], elaborou-se a tabela 2 que sintetiza a analise de trabalhos sobre TIS ([4] [6] [5] [7] [8] [9] [10]). Dos trabalhos analisados, apenas um apresenta um framework computacional. A figura 2 representa a proposta dos autores. De um modo geral, o framework mostra quatro elementos: perspectiva local, o framework de modelagem de grupos (composto por um Classificador de interação, Aprendizado de Colaboração e Detecção Evolucionária de Identidades Grupais) e uma perspectiva global. Tendo como base o trabalho de [7] uma proposta inicial para a implementação do classificador de interação é apresentada na próxima seção. Para trabalhos futuros pretende-se implementar os outros módulos para que se possa fazer uma analise mais profunda em relação aos conceitos da TIS e do framework proposto.

Tabela 1: Comparativo de modelos elaborado por [23]

\begin{tabular}{|l|l|l|l|}
\hline Modelo & Tipos de Identidade & Fonte de dados & Questões chave \\
\hline SCIPR - [6] & Flexível: Opiniões políticas & $\begin{array}{l}\text { Dados IRA de ataque e re- } \\
\text { sultados de votos da Irlanda } \\
\text { do Norte }\end{array}$ & $\begin{array}{l}\text { Modelos de influencia utili- } \\
\text { zando um modelo de confi- } \\
\text { ança limitado. Inclui múlti- } \\
\text { plas identidades sobrepostas } \\
\text { e usa uma rede social sim- } \\
\text { ples para influencia. }\end{array}$ \\
\hline $\begin{array}{l}\text { Modelo de Meta- } \\
\text { contraste de Sal- } \\
\text { zarulo - [27] }\end{array}$ & $\begin{array}{l}\text { Flexível: Categorias sociais } \\
\text { baseadas em crenças }\end{array}$ & Sintéticos & $\begin{array}{l}\text { Ilustra como a polarização } \\
\text { e o extremismo pode ocor- } \\
\text { rer devido a combinação de } \\
\text { atração para ingroups e re- } \\
\text { pulsa a outgroups. }\end{array}$ \\
\hline PS-I - [4] & $\begin{array}{l}\text { Flexível e descendente: } \\
\text { identidade grupal baseada } \\
\text { em cultura/politica }\end{array}$ & $\begin{array}{l}\text { Expertise regional do autor } \\
\text { Clusters de modelos geo- } \\
\text { gráficos, ou de politicas, } \\
\text { espalha-se as identidades } \\
\text { pela população. }\end{array}$ \\
\hline SILAS - [23] & $\begin{array}{l}\text { Ética, religiosa e identidade } \\
\text { de politica de afiliação }\end{array}$ & $\begin{array}{l}\text { Modelos de como conflitos } \\
\text { entre identidades podem ser } \\
\text { resolvidos; Modelos de di- } \\
\text { nâmica de inimigo em co- } \\
\text { mum. }\end{array}$ \\
\hline
\end{tabular}

Figura 2: Framework computacional elaborado por [7]

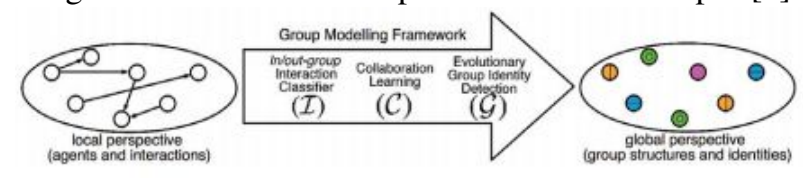

Revista Brasileira de Computação Aplicada (ISSN 2176-6649), Passo Fundo, v. 9, n. 1, p. 73-83, abr. 2017 
Tabela 2: Síntese de trabalhos que abordam a Teoria da Identidade Social

\begin{tabular}{|c|c|c|}
\hline Trabalho & Tema & Contribuiçãa \\
\hline [4] & $\begin{array}{l}\text { Plataforma de simulação baseada em agen- } \\
\text { tes originalmente desenvolvida para opera- } \\
\text { cionalizar, refinar, e testar versões concor- } \\
\text { rentes da teoria da identidade construtivista }\end{array}$ & Plataforma de simulação \\
\hline [6] & $\begin{array}{l}\text { São simuladas identidades culturais para } \\
\text { prever reações a eventos }\end{array}$ & Modelo computacional \\
\hline [5] & $\begin{array}{l}\text { Os autores discutem o papel de agentes in- } \\
\text { teragindo em situações sociais complexas } \\
\text { dos quais necessitam levar em conta o con- } \\
\text { texto social para desenvolver confiança }\end{array}$ & $\begin{array}{l}\text { Trata-se de um resumo es- } \\
\text { tendido, onde apenas é re- } \\
\text { forçada a ideia da TIS em } \\
\text { SMA }\end{array}$ \\
\hline [7] & $\begin{array}{l}\text { É apresentado um framework computaci- } \\
\text { onal capaz de inferir a existência de gru- } \\
\text { pos, construídos em redes sociais de ami- } \\
\text { zades recíprocas, em uma Sociedade Arti- } \\
\text { ficial Adaptativa Complexa }\end{array}$ & Framework computacional \\
\hline [8] & $\begin{array}{l}\text { Sistema baseado em agentes para simular a } \\
\text { dinâmica de crenças de identidade social. }\end{array}$ & Sistema baseado em agentes \\
\hline [9] & $\begin{array}{l}\text { Modelo baseado em agentes para a dinâ- } \\
\text { mica da identidade social }\end{array}$ & Modelo baseado em agentes \\
\hline [10] & $\begin{array}{l}\text { Modelo dinâmico de identidade para agen- } \\
\text { tes do qual promove agentes com iden- } \\
\text { tidade adaptativa e seu comportamento é } \\
\text { ajustável ao contexto social. }\end{array}$ & Modelo dinâmico \\
\hline [28] & $\begin{array}{l}\text { Influencia da presença social na participa- } \\
\text { ção continua online em comunidades vir- } \\
\text { tuais, na visão relacional baseada na teoria } \\
\text { da identidade social. }\end{array}$ & Dois modelos de pesquisa \\
\hline [29] & $\begin{array}{l}\text { Concentra-se em conceitos de consciência } \\
\text { social para dar suporte a computação social }\end{array}$ & $\begin{array}{l}\text { Conceitos sociais para si- } \\
\text { mulação de interação entre } \\
\text { agentes }\end{array}$ \\
\hline [23] & $\begin{array}{l}\text { Elucida a modelagem de identidade so- } \\
\text { cial, contextualizando trabalhos passados e } \\
\text { questões relevantes na modelagem socio- } \\
\text { cultural }\end{array}$ & Elenco de quatro modelos \\
\hline
\end{tabular}

\section{Proposta - um classificador de interação}

Baseado na figura 1 apresentada na seção de estrutura das TS, compreende-se as trocas como relações. [30] consideram essas relações como a existência ou inexistência de uma certa conexão entre pares de objetos assumidos em uma determinada ordem. Formalmente, é definida uma relação em termos de "pares ordenados", onde um par ordenado de elementos $a$ e $b$, onde $a$ é designado como o primeiro elemento e $b$ como segundo elemento, então denotado por $(a, b)$. Uma forma de visualizar uma relação $R$ em um conjunto finito é primeiramente escrever os elementos do conjunto e, então, desenhar uma flecha de cada elemento $x$ para cada elemento $y$, sempre que $x$ estiver relacionado com $y$. Esse diagrama é chamado grafo orientado da relação. A figura 3 mostra o grafo orientado da seguinte relação $\mathrm{R}=\{(1,2),(2,2),(2,4),(3,2),(3,4),(4,1),(4,3)\}$ sob o conjunto $\mathrm{A}=\{1,2,3,4\}$. É observado que há uma flecha de 2 nele mesmo, uma vez que 2 é relacionado a $2 \operatorname{sob} R$.

Tendo em vista as relações, considera-se um conjunto $S$ não vazio. Uma relação $R$ sob $S$ é uma relação de equivalência se $R$ é reflexiva, simétrica e transitiva. Ou seja, $R$ é uma relação de equivalência sobre $S$ se tiver as três propriedades a seguir: 
Figura 3: Grafo orientado da relação de [30]

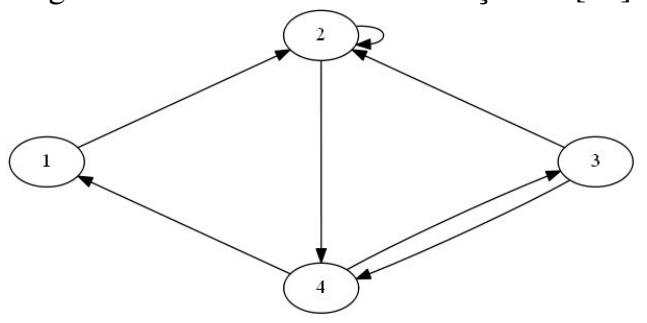

1. Para todo $a \epsilon S, a R a$.

2. Se $a R b$, então $b R a$.

3. Se $a R b$ e $b R c$, então $a R c$.

A ideia geral por trás de uma relação de equivalência é a de que se trata de uma classificação de objetos que são, de algum modo, "semelhantes". De fato, a relação "="de igualdade sobre qualquer conjunto $S$ é uma relação de equivalência, ou seja:

1. $a=a$ para todo $a \epsilon S$.

2. Se $a=b$, então $b=a$.

3. Se $a=b, b=c$, então $a=c$.

Tratando-se da relação de equivalência para pares ordenados, determina-se uma TS como uma relação de equivalência. Considerando um conjunto com três elementos $C=\{1,2,3\}$, onde esses elementos são agentes em uma troca social, tem-se o seguinte grafo orientado da relação na figura 4.

Figura 4: Grafo Orientado de uma TS entre três agentes

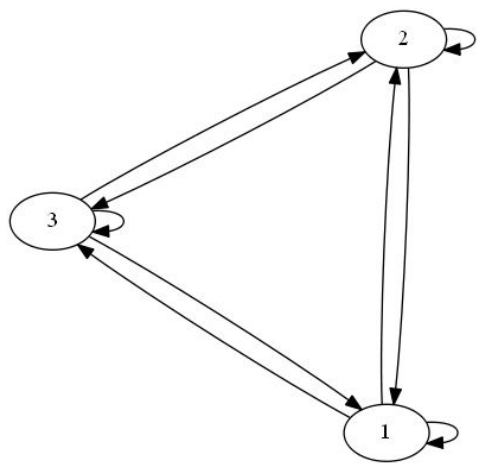

Considerando os três agentes do conjunto $\mathrm{C}=\{1,2,3\}$, as flechas indicam uma troca social, podendo ser de sucesso ou não. Ressalta-se que aqui a propriedade da relação de equivalência reflexiva não se aplica, pois consideram-se as TS entre pares de agentes diferentes. Partindo desse princípio e considerando uma rede de agentes interagindo (aqui é importante considerar que o sistema já executou as trocas sociais tendo como base ciclos de execução, por exemplo), têm-se uma estrutura de sociedade similar a figura 5, apresentada em [13].

Pela própria distribuição da rede, é possível visualizar estruturas grupais. Contudo, sem considerar aspectos sociais e de identidade. Nossa proposta baseia-se primeiramente na classificação dessas interações tendo como base trocas de sucesso entre pares de agentes. Para isso, é necessário, como ponto de partida, a perspectiva local que no caso do JAPTS baseia-se em ciclos onde os agentes tentam interagir. A rede apresentada na figura 5 tem algum numero especifico de ciclos para sua construção final, esses ciclos são registrados em cenários de teste que 
Figura 5: Rede de interações entre agentes de [13]

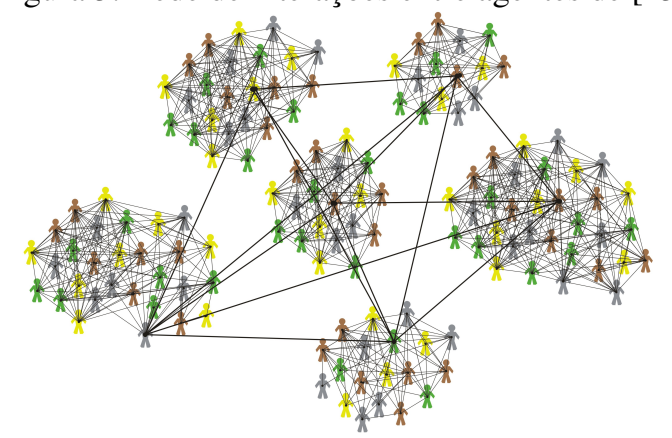

serão elaborados. Para a segunda etapa de classificação de interação, espera-se a reestruturação original da rede (que considera apenas as trocas, sendo de sucesso ou não), ou seja, readequando a rede é possível observar diferentes estruturas de interação entre os agentes, onde será possível observar os grupos considerando suas estratégias (egoísta, racional e altruísta), por exemplo. Tendo essa reestruturação da rede com base nas questões levantadas, a terceira etapa envolve um modulo de Aprendizado de Colaboração ([7]) onde os níveis de colaboração dos agentes são identificados, analisados e reestruturados. Essas duas etapas ainda serão implementadas.

Sejam dois agentes $i$ e $j$. Para cada agente é definida uma estratégia de jogo $r, r^{\max }$ e $s^{\min } \in[0 ; 1]$, investimento, investimento máximo e satisfação mínima, respectivamente. São parâmetros da estratégia de troca social dada a cada agente. Logo, em um jogo entre os dois agentes $i$ e $j$ com o agente $i$ com a seguinte estratégia $\left(r_{i}, r_{i}^{\max }, s_{i}^{\min }\right)$ e o agente $j$ com a respectiva estratégia $\left(r_{j}, r_{j}^{\max }, s_{j}^{\min }\right)$.

Seja $r_{i}$ o investimento realizado pelo agente $i$ e $s_{j}^{m i n}$ a menor satisfação aceitável de $j$. Ocorrerá a troca de $i \operatorname{com} j$ se a oferta realizada por $i$ for maior ou igual a satisfação mínima aceitável de $j$, ou seja, ocorre a troca entre $i$ e $j$.

O processo de trocas entre dois agentes é mostrado na Figura 6 que considera as diferentes estratégias de troca social apresentadas em [13], e definidas para a análise deste modelo.

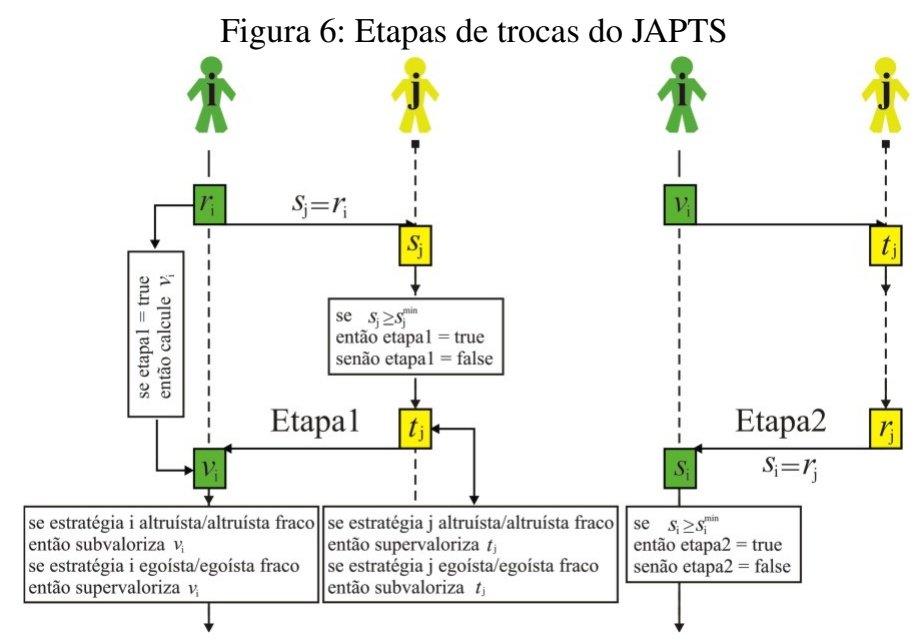

Portanto, o classificador de interação teria a seguinte estrutura apresentada na figura 7.

Observa-se que nessa proposta existirá apenas dois grupos: agentes com trocas sociais de sucesso $\mathrm{X}$ agentes com trocas sociais que falharam. Essa classificação é análoga as identificações ingroup e outgroups, originárias da TIS. Contudo, o módulo de aprendizado de colaboração irá identificar novas estruturas de colaboração nos relacionamentos entre agentes e novos cenários poderão ser identificados. 
Figura 7: Proposta do classificador de interação

\section{Classificador de Interação}

Se propriedades da relação de equivalência Ok, então:

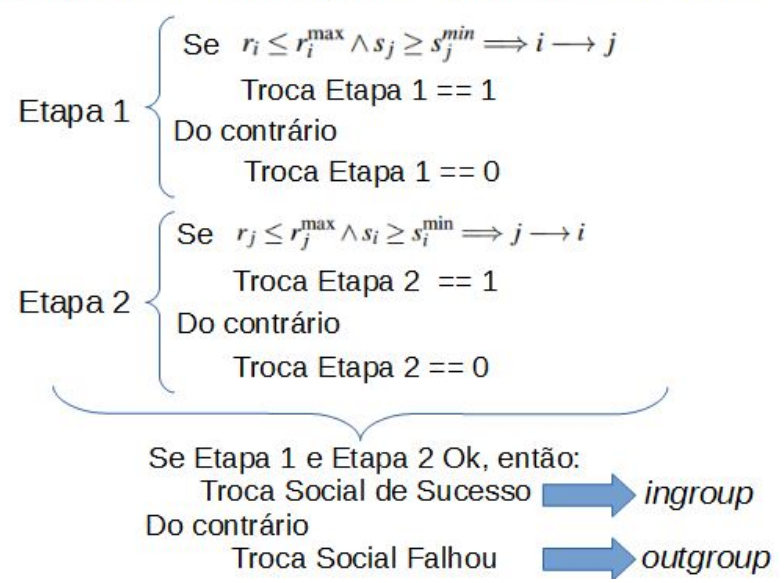

\section{Conclusões parciais}

Inicialmente [2] [1] e seus colaboradores propuseram estudos de experimentação intergrupal questionando se a discriminação poderia estar ligada a conflitos sociais ou mesmo a algum histórico de hostilidade. $\mathrm{O}$ autor concluiu que o simples fato de uma divisão em grupos poderia disparar um comportamento discriminatório. Estas pesquisas iniciais motivaram o desenvolvimento da Teoria da Identidade Social (TIS). A TIS vem sendo pesquisada dentro da esfera de SMA, contudo sem abordar sistemas de Trocas Sociais. Um dos pontos de contribuição é a autorregulação desses processos, onde é abordado pelo JAPTS em [13]. Motivados pela incorporação da TIS e do JAPTS foi desenvolvido um modelo inicial do experimento original conduzido por [1]. Este artigo apresentou o referencial teórico desta pesquisa, bem como trabalhos da área de SMA que desenvolveram esforços na integração entre os conceitos da psicologia social e inteligência artificial. Tendo como base a revisão de literatura, observou-se a existência de um framework computacional capaz de inferir estruturas grupais em uma sociedade de agentes. Para este momento, apresentou-se uma proposta inicial para a divisão inicial de grupos considerando um conceito inicial da TIS. Para trabalhos futuros pretende-se implementar a proposta apresentada, assim como os outros módulos propostos no framework dos autores. Com isso, espera-se que a sociedade decorrente do JAPTS seja analisada considerando aspectos humanos cada vez mais próximos do mundo real.

\section{Agradecimentos}

Os autores desta pesquisa gostariam de agradecer a agencia financiadora CAPES e o Centro de Ciências Computacionais - C3 - e ao Laboratório de Sistemas Multiagentes e Simulação Social e Ambiental (LAMSA) da Universidade Federal do Rio Grande por proverem os recursos necessários para desenvolver esta pesquisa.

\section{Referências}

[1] TAJFEL, H. et al. Social categorization and intergroup behaviour. European Journal of Social Psychology, Wiley Online Library, v. 1, n. 2, p. 149-178, 1971.

[2] TAJFEL, H. Experiments in intergroup discrimination. Scientific American, v. 223, n. 5, p. 96-102, 1970.

[3] FERREIRA, M. C. A Psicologia Social contemporanea: principais tendencias e perspectivas nacionais e internacionais. Psicologia: Teoria e Pesquisa, scielo, v. 26, p. 51 - 64, 00 2010. ISSN 0102-3772. Disponí-

Revista Brasileira de Computação Aplicada (ISSN 2176-6649), Passo Fundo, v. 9, n. 1, p. 73-83, abr. 2017 
vel em: <http://www.scielo.br/scielo.php?script=sci_arttext\&pid=S0102-37722010000500005\&nrm=iso $>$. Acesso em: 30 abr. 2017.

[4] LUSTICK, I. Ps-i: A user-friendly agent-based modeling platform for testing theories of political identity and political stability. Journal of Artificial Societies and Social Simulation, v. 5, n. 3, 2002.

[5] PRADA, R. et al. The role of social identity, rationality and anticipation in believable agents. In: INTERNATIONAL FOUNDATION FOR AUTONOMOUS AGENTS AND MULTIAGENT SYSTEMS. Proceedings of the 11th International Conference on Autonomous Agents and Multiagent Systems-Volume 3. [S.1.], 2012. p. 1175-1176.

[6] GRIER, R. A. et al. Scipr: A computational model to simulate cultural identities for predicting reactions to events. In: Proceedings of the Second International Conference on Computational Cultural Dynamics. [S.1.: s.n.], 2008.

[7] GRAPPIOLO, C.; TOGELIUS, J.; YANNAKAKIS, G. N. Artificial evolution for the detection of group identities in complex artificial societies. In: IEEE. Artificial Life (ALIFE), 2013 IEEE Symposium on. [S.1.], 2013. p. 126-133.

[8] UPAL, M. A.; GIBBON, S. Agent-based system for simulating the dynamics of social identity beliefs. In: Proceedings of the 48th Annual Simulation Symposium. San Diego, CA, USA: Society for Computer Simulation International, 2015. (ANSS '15), p. 94-101. ISBN 978-1-5108-0099-1. Disponível em: <http://dl.acm.org/citation.cfm?id=2876341.2876354>. Acesso em: 30 abr. 2017.

[9] SMALDINO, P. et al. An agent-based model of social identity dynamics. Journal of Artificial Societies and Social Simulation, v. 15, n. 4, p. 7, 2012.

[10] DIMAS, J.; PRADA, R. You are who you hang out with: Agents with dynamic identity. In: INTERNATIONAL FOUNDATION FOR AUTONOMOUS AGENTS AND MULTIAGENT SYSTEMS. Proceedings of the 2013 international conference on Autonomous agents and multi-agent systems. [S.1.], 2013. p. 13371338 .

[11] PEREIRA, D. R. Construção de planos BDI a partir de políticas ótimas de POMDPs, com aplicação na auto-regulação de trocas sociais em sistemas multiagentes. 2008.

[12] GONÇALVES, L. V. Uma arquitetura de Agentes BDI para auto-regulação de Trocas Sociais em Sistemas Multiagentes Abertos. 2009.

[13] MACEDO, L. Uma Abordagem Evolucionária e Espacial para o Jogo da Autorregulação de Processos de Trocas Sociais em Sistemas Multiagentes. Dissertação (Mestrado) - Programa de Pós-graduação em Computação da Universidade Federal do Rio Grande, 2013.

[14] LAER, A. G. von. Autorregulação de Processos de Trocas Sociais em SMA: um modelo de sociedade de agentes BDI evolucionários e culturais no contexto do JaCaMo. Dissertação (Mestrado) - Programa de Pós-graduação em Computação da Universidade Federal do Rio Grande, 2014.

[15] ROJAS, Y. Trocas Sociais em Sistemas Multiagentes: Transferência de Confianção com Base na Reputação e na Relação de Dependência. Dissertação (Mestrado) - Programa de Pós-graduação em Computação da Universidade Federal do Rio Grande, 2015.

[16] SALDANHA, J. et al. Modelagem da teoria da identidade social em sistemas multiagente. In: WESAAC. Anais do X Workshop-Escola de Sistemas de Agentes, seus Ambientes e apliCacoes WESAAC 2016. [S.1.], 2016. p. 187-192.

[17] TAJFEL, H. Human groups and social categories: Studies in social psychology. [S.1.]: CUP Archive, 1981.

[18] TAJFEL, H.; TURNER, J. C. The social identity theory of intergroup behavior. Political psychology: Key readings. Key readings in social psychology., (pp. 276-293), Psychology Press, 2004. 
[19] BROWN, R. Social identity theory: Past achievements, current problems and future challenges. European journal of social psychology, Wiley Online Library, v. 30, n. 6, p. 745-778, 2000.

[20] HOGG, M. A. Social identity theory. Contemporary social psychological theories, v. 13, p. 111-1369, 2006.

[21] PIAGET, J. Sociological studies. London: Routlege, 1995.

[22] DIMURO, G. P.; COSTA, A. C. da R. Exchange values and self-regulation of exchanges in multi-agent systems: The provisory, centralized model. In: BRUECKNER, S. et al. (Ed.). Engineering Self-Organising Systems. Springer, 2005. (Lecture Notes in Computer Science, v. 3910), p. 75-89. ISBN 3-540-33342-8. Disponível em: <http://dx.doi.org/10.1007/11734697_6>. Acesso em: 30 abr. 2017.

[23] KOPECKY, J.; BOS, N.; GREENBERG, A. Social identity modeling: past work and relevant issues for socio-cultural modeling. In: Proceedings of the 19th Conference on Behavior Representation in Modeling and Simulation, Charleston, SC (pp. 203-210). [S.1.: s.n.], 2010.

[24] REID, F. Rediscovering the social group: A self-categorization theory. British Journal of Social Psychology, Wiley Online Library, v. 26, n. 4, p. 347-348, 1987.

[25] BRUNER, J. S. On perceptual readiness. Psychological review, American Psychological Association, v. 64, n. 2 , p. $123,1957$.

[26] BLANZ, M. Accessibility and fit as determinants of the salience of social categorizations. European Journal of Social Psychology, Wiley Online Library, v. 29, n. 1, p. 43-74, 1999.

[27] SALZARULO, L. A continuous opinion dynamics model based on the principle of meta-contrast. Journal of Artificial Societies and Social Simulation, v. 9, n. 1, 2006.

[28] WANG, K.; TAI, C.-F. The influence of social presence on continual participation in online communities: The relational view based on social identity theory. In: IEEE. Service Sciences (IJCSS), 2011 International Joint Conference on. [S.1.], 2011. p. 110-115.

[29] RAKOTONIRAINY, A.; LOKE, S. W.; OBST, P. Social awareness concepts to support social computing. In: IEEE. Computational Science and Engineering, 2009. CSE'09. International Conference on. [S.1.], 2009. v. 4, p. 223-228.

[30] LIPSCHUTZ, S.; LIPSON, M. Matemática Discreta-: Coleção Schaum. [S.1.]: Bookman Editora, 2013. 